\title{
Flujos y Reflujos en el Desarrollo del Budismo Asiático: un Recorrido por la Historia del Movimiento de Meditación Vipassaná
}

\author{
Fluxos e Refluxos no Desenvolvimento do Budismo Asiático: \\ um Percurso pela História do Movimento de Meditação de Vipassana
}

Flows and Ebbs in the Development of Asian Buddhism: a Journey through the History of the Vipassana Meditation Movement

Catón Eduardo Carini

RESUMO

El presente artículo se centra en indagar en la historia de la organización budista internacional Vipassana Meditation. Primero, realiza un recorrido por el desarrollo del budismo en Asia, haciendo hincapié en su afincamiento en Birmania. Luego, explora las trayectorias vitales de los líderes religiosos que formaron la mencionada organización y analiza los principales rasgos de la cosmovisión vipassana. Finalmente, reflexiona sobre la forma en que el budismo se desarrolla mediante un proceso de flujo de ideas y prácticas que van y vienen entre la India, el Sudeste Asiático y Occidente.

PALAVRAS-CHAVE: Vipassana. Budismo. Historia. Globalización.

ABSTRACT

This article focuses on the history of the international Buddhist organization known as Vipassana Meditation. First, it explores the development of Buddhism in Asia, placing particular emphasis on its position in Burma. Then it looks at the life trajectories of the religious leaders who created the organization and analyzes the main features of the vipassana worldview. Finally, it reflects on the development of Buddhism through a process of ideas and practices flowing back and forth between India, Southeast Asia and the West.

KEYWORDS: Vipassana. Buddhism. History. Globalization.

\footnotetext{
1 Recebido em 30/05/2017. Aprovado em 30/09/2017.

2 Doutor em Antropología. Professor de Antropología da Universidad Nacional de La Plata, Argentina. Email: catoncarini@yahoo.com.ar.
} 


\section{INTRODUCCIÓN}

El presente trabajo indaga el proceso histórico de desarrollo de una organización budista internacional, Vipassana Meditation, fundada por Satya Narayan Goenka. Esta institución originada en Birmania a mediados del siglo pasado se inscribe dentro de la tradición del budismo theravada que, junto al mahayana y el vajrayana, designa a una de las tres grandes ramas del budismo. Su rasgo fundamental es que se centra en la práctica del vipassana ("visión cabal"), una técnica de meditación descripta en Satipatthana Sutta del Canon Pali, la recopilación de los discursos del Buda.

Vipassana Meditation es conocido por muchas personas que transitan por espacios de la espiritualidad oriental. El primer paso a dar en el mundo de la meditación vipassana se conforma por la asistencia a sus cursos de 10 días de duración. Cualquier persona que quiera iniciarse en esta práctica deberá realizar un curso completo como "estudiante nuevo". Una vez finalizada esta primera etapa el mismo pasará a convertirse en "estudiante antiguo", lo cual lo habilitará a participar de todas las demás actividades y a acceder a la sección del sitio web reservada para los "antiguos".

Los centros de Vipassana Meditation en donde se realizan estos cursos generalmente se encuentran emplazados en espacios suburbanos, con grandes áreas de parque que permiten a los estudiantes estar en contacto con la naturaleza. Los asistentes, en su mayoría, son personas sin experiencia previa en meditación, de clase media, de entre 20 y 40 años de edad, habiendo una fuerte presencia de personas dedicadas a las humanidades y las artes, la psicología y las terapias alternativas. Muchos tienen una trayectoria religiosa signada por el desencanto con la religión heredada familiarmente - en su mayor parte católica -, y el posterior tránsito por diversos grupos vinculados a la espiritualidad oriental o la nueva era.

Estos cursos presentan algunas características particulares que llaman la atención de la persona que busca en la oferta religiosa una alternativa para aprender meditación. Uno de los rasgos que quizás más impacte es que los cursos se realizan en completo silencio y 
no se permite el contacto físico entre ninguna persona, ni siquiera el contacto visual ${ }^{3}$. Además, la práctica de meditación sigue un horario riguroso que comienza al despertar la las cuatro de la mañana) y termina a la hora de dormir (a las nueve y media de la noche). Durante el día, la jornada se estructura sobre un ajustado cronograma que se basa en diez u once horas de meditación distribuidas en cuatro "bloques", con intervalos para la alimentación y el descanso ${ }^{4}$. Por otro lado, existe una completa segregación del mundo exterior. Durante la totalidad del curso no está permitido ningún tipo de visita o el uso del teléfono móvil. Tampoco está permitida la lectura, la escritura, escuchar música, sacar fotos o grabar audios. Además, se pide a los participantes que se abstengan de realizar rituales religiosos, rezar o llevar cualquier tipo de objeto sagrado tales como cruces, rosarios, incluso malas (rosarios budistas). También se les solicita suspender cualquier práctica espiritual, energética o física, tales como correr, yoga, taichi, reiki, recitación de mantras, visualización o cualquier otra forma de meditación. En suma, durante el período de retiro el participante restringe todas sus actividades religiosas, relaciones sociales y pasatiempos cotidianos con el fin explícito de dedicar toda su atención y esfuerzo a aprender y practicar la técnica de vipassana.

Los cursos son completamente gratuitos y se sostienen exclusivamente por las donaciones de los "estudiantes antiguos", es decir, quienes ya han participado en algún curso de 10 días. Por otro lado, no se acepta dinero de nadie que no haya realizado al menos uno de estos cursos. Recién luego de haber realizado un curso de 10 días inmediatamente después o cuando se desee - que se puede efectuar la donación de forma anónima y con el propósito de financiar futuros cursos ${ }^{5}$. Finalmente, existe un estricto "código de disciplina" que todo participante debe cumplir. Este código se estructura en cinco preceptos, como ser la abstención de matar cualquier ser vivo (incluyendo insectos

\footnotetext{
${ }^{3}$ Aunque el participante puede hablar con el "manager" designado para el curso para resolver cuestiones de orden práctico u organizativo, o con el maestro o maestra que dirige el curso en determinados horarios estipulados, para consultas sobre la práctica de la meditación.

${ }^{4}$ Es preciso realizar el curso completo, durante la totalidad de los 10 días, y se requiere un compromiso total para terminarlo, aunque es frecuente que se retiren algunos participantes a lo largo del curso. En base a nuestra experiencia en cuatro cursos, cerca de $5 \%$ de los participantes abandonaron cada vez por diferentes motivos.

${ }^{5}$ Se fomenta la idea de que las personas que toman un curso lo hacen gracias a las donaciones efectuadas por otros meditadores, de modo que si uno considera beneficioso lo aprendido, tiene la obligación implícita de realizar un contra-don para brindar a otros la posibilidad de beneficiarse aprendiendo gratuitamente la técnica de vipassana en futuros cursos.
} 
tales como hormigas y mosquitos), de robar, mentir, de cualquier tipo de actividad sexual y del consumo de intoxicantes (incluídos el alcohol, tabaco, pastillas para dormir etc.]

Pese a esta suma de restricciones, la práctica del vipassana convoca a cada vez más participantes, atraídos por la posibilidad de aprender meditación de la mano de una organización que ha ganado renombre internacional en tanto difusora de la técnica de meditación más cercana a la propuesta por el Buda. En la actualidad, la organización Vipassana Meditation cuenta con 308 centros distribuidos en 94 países de África, América, Asia, Europa y Oceanía. Más de la mitad de ellos, unos 174, son espacios residenciales propios adquiridos por donaciones voluntarias ${ }^{7}$. En el mundo, el volumen de asistentes a los cursos de vipassana rondan las 120.000 personas cada año. Con frecuencia las vacantes para participar se acaban varias semanas o meses antes. Como hay más demanda que oferta de espacios disponibles es imprescindible confirmar la asistencia al curso 10 días antes y en el caso de no poder, el participante se compromete a avisar hasta el último minuto para así poder dar su lugar a aquellos interesados que aún permanecen "en lista de espera"

¿Cuáles son los elementos que propician la pregnancia de una práctica meditativa que, a primera vista, ofrece una experiencia tan ajena al habitus occidental? ¿Qué factores influyeron en que el vipassana adquiera un alcance global cuando, incluso en Asia, hasta hace pocas décadas, era una práctica poco común? Como sugiere Baumann (2001), para comprender el proceso de expansión del budismo en Occidente hace falta tener en cuenta no sólo la historia y el presente de esta religión en Europa y América, sino también los cambios y desarrollos en Asia. Al mismo tiempo, no hay olvidar que muchos de esos cambios se debieron al influjo de Occidente en el budismo asiático, sobre todo de la mano de la colonización y la modernidad, configurando una vía de doble mano de mutua influencia por la cual circulan ideas, conceptos y prácticas.

\footnotetext{
${ }^{6}$ Existe una completa segregación de los sexos, con dormitorios, sanitarios, duchas, comedor y espacios de esparcimiento separados. Si bien la sala de meditación es compartida, se dispone entradas separadas para hombres y mujeres, los cuales se sientan a uno u otro lado de la habitación. Además la comida es vegetariana y no es posible llevar alimentos al centro.

7 Además de los cursos de 10 días, también se ofrecen "cursos cortos" de 1 a 3 días y "cursos largos" de 20, 30,45 y 60 días, todos ellos para "estudiantes antiguos".

${ }^{8}$ En este artículo no realizamos una descripción exhaustiva de la técnica de meditación vipassana ni nos centramos en indagar los aspectos experienciales, corporales e intersubjetivos de esta práctica. Al respecto, se sugiere consultar los interesantes aportes de Carvalho (2013), Eddy (2012), Pagis $(2010,2015)$ y Sharf (1995).
} 
Por ello, a continuación examinaremos los antecedentes que hicieron posible la formación de la organización internacional Vipassana Meditation, desde su origen en la India, pasando por su enraizamiento en Birmania y su expansión por todo el Occidente.

\section{LA DIFUSIÓN DEL BUDISMO EN EL SUDESTE ASIÁTICO Y EL MOVIMIENTO VIPASSANA}

El budismo nació en la India hace 2.500 años. Dos siglos después, el emperador Asoka, gobernante de gran parte del subcontinente Indio, se convirtió al budismo y lo difundió por todo su reinado. En esa época comenzó su difusión hacia el Extremo Oriente. Asia Central y Oriental recibieron la influencia de las ramas del budismo mahayana y vajrayana, mientras que en el Sudeste Asiático prosperó la rama theravada, considerada como la más cercana al budismo inicial en su doctrina y sus prácticas. Un lugar neurálgico que ha jugado un rol clave en la difusión del budismo en el Sudeste Asiático es la isla de Ceilán. Aquí el budismo llega en el siglo III a.C. desde la India de la mano de misioneros enviado por Asoka. Ceilán ha sido por más de dos milenios una usina clave a la hora de propagar o revitalizar el budismo en el resto del Asia Sudoriental, incluyendo Birmania, Laos, Tailandia y Camboya.

Con respecto a Birmania, este país ha sido siempre un baluarte importante del budismo theravada en el Lejano Oriente, ya que muchas veces funcionó para revitalizar esta religión en todo el continente. Mantuvo con la India relaciones comerciales desde hace milenios por medio de rutas marítimas y terrestres (Smith, 1963; Harvey, 1998). Según afirma Goenka (2011), la primera Ilegada del budismo a Birmania se produjo en los inicios de esta religión, cuando dos hermanos comerciantes originarios de la India, Tapassu y Bhallika, volvían de Birmania y se encontraron con el Buda meditando en el bosque luego de su iluminación. Le ofrecieron una comida, la primera que tomaba el Buda en semanas, a la cual retribuyó ofreciendo ocho cortes de su propio cabello. Los hermanos volvieron a Birmania, donde ofrecieron las reliquias al rey de Ukkalapati, quien las entronizó en tres pagodas construidas para ese fin - entre ellas la famosa Pagoda Shwedagon -. La segunda vez que el budismo llegó a Birmania, según Goenka, fue debido al viaje de Gavampati, un destacado discípulo del Buda, quien viajó a la ciudad de Suvannabhumi unos ocho años después de la muerte de su maestro para enseñarle al gobernante de aquella ciudad, 
Siharaja. La tercera, fue durante el período del emperador Asoka en el siglo III a.C, quien mandó dos monjes misioneros, Sona y Uttara a Birmania que formaron una pequeña comunidad budista (Goenka 2011; Harvey, 1998; Smith, 1963).

A partir de ese momento Birmania recibió un influjo budista constante, principalmente de Ceilán. Hay huellas arqueológicas del budismo theravada en el siglo V, aunque no fue sino hasta el siglo XI que el rey Anoratha convirtió a todo el país a esta religión. Desde el siglo XV quedó establecido por el rey Dhammaceti un vínculo oficial con Ceilán en tanto fuente de legitimación ortodoxa de la orden monástica birmana. Esta relación de patronazgo y protección de la comunidad monástica por parte del poder político es una constante a lo largo de la historia del budismo en Birmania. Desde los inicios de esta religión todos los reyes construyeron templos, reprodujeron copias del Canon Pali y edificaron pagodas. El último de los monarcas defensores del budismo fue el rey Mindon, quien en el siglo XIX organizó el $V$ Concilio Budista en el cual se trascribió la totalidad del Canon Pali en 729 losas de piedra (Bareau, 1981; Harvey, 1998; Smith, 1963)

Después de la ocupación británica en 1885, los monjes por lo general actuaron como activistas por la independencia, criticando la dominación colonial. Cuando esta independencia se produjo, en el año 1948, el budismo volvió a tener el apoyo político, ya que el presidente $\mathrm{U} \mathrm{Nu}$ creía que una versión socialista y nacionalista del estado posibilitaría alcanzar la meta budista de una sociedad más justa y pacífica. Bajo el auspicio de este gobernante se realizó en 1954 el VI Concilio Budista en Rangún, la capital del país. En 1962 un golpe de estado instauró un gobierno militar con algunos rasgos marxistas, cuya cabeza era el general Ne Win. Si bien este gobierno no era hostil al budismo, y de hecho su aislacionismo lo favoreció, recibió una activa movilización de la comunidad monástica debido a sus medidas autoritarias y su ineficiencia para combatir la pobreza (Smith, 1963) .

Actualmente en Birmania casi el $90 \%$ de la población es budista, siendo oficialmente considerada como la religión de estado. La comunidad monástica goza de prestigio social y se sustenta en base a las donaciones de los laicos, que acuden a ellos en busca de, entre otras cosas, educación alfabetizada para sus hijos, conjurar todo tipo de desgracias, recibir adivinaciones, alcanzar el éxito económico, la salud o la felicidad. En

\footnotetext{
${ }^{9}$ Este gobierno culminó en 1988 cuando se produjeron una serie de incidentes por la protesta de un amplio sector de la población, en la cual fueron reprimidos y asesinados por el ejército miles de personas, entre las cuales se encontraban estudiantes universitarios, monjes budistas e intelectuales críticos.
} 
Flujos y Reflujos en el Desarrollo del Budismo Asiático:

un Recorrido por la Historia del Movimiento de Meditación Vipassana

suma, los monjes son considerados una fuente de protección, de poder sobrenatural, y reciben a cambio el sustento material para sus comunidades. La vida cotidiana de los monjes no necesariamente implica la práctica de la meditación. En general se centra en la realización de ceremonias para los laicos, incluyendo ritos de paso que tramiten momentos importantes de la vida de los fieles, tales como el nacimiento, el casamiento y la muerte, junto al estudio y la recitación de los textos canónicos del budismo (Bareau, 1981).

Pero paralelamente a este budismo tradicionalista ${ }^{10}$, centrado en el estudio intelectual y en las ceremonias para los fieles laicos, desde fines del siglo XIX y principios del $\mathrm{XX}$ se ha estado desarrollando el budismo modernista o reformista, que cuestiona la postura del budismo tradicionalista en tanto no es posible obtener la iluminación en esta era". El budismo reformista en Birmania dio lugar al denominado "Movimiento Vipassana". Como su nombre lo evidencia, el Movimiento Vipassana de budismo reformista se caracteriza por la importancia otorgada a meditación vipassana en menoscabo de la práctica de la concentración (samatha). Su postura enfatiza que se puede practicar el vipassana con un grado de concentración básico, accesible al laicado en un periodo de tiempo relativamente corto. Atraviesa varios países del Sudeste Asiático tales como Laos, Ceilán, Tailandia y Birmania, donde surgieron diversas tradiciones, tales como la "Tradición Tailandesa del Bosque", que apuntaban contra el intelectualismo monástico hegemónico.

En Birmania las dos vertientes más importantes del Movimiento Vipassana son el "Nuevo Método Birmano", creado por U Narada y popularizado por Mahasi Sayadaw, el cual tuvo una gran difusión en Estados Unidos de la mano de maestros occidentales tales como Jack Kornfield, y el linaje de Ledi Sayadaw, el cual dio origen a la organización

\footnotetext{
${ }^{10}$ El budismo tradicionalista o histórico comienza con el reinado de Asoka y continúa hasta la actualidad. Su rasgo más importante es que concibe la meta soteriológica de la iluminación "muchas vidas adelante", después de un largo camino de purificación de las imperfecciones kármicas. De modo que con este tipo de budismo adquieren cada vez mayor importancia otras prácticas diferentes de la meditación tales como el ganar merito a través de rituales u ofrendas para obtener cosas inmediatas para la vida cotidiana y un buen renacimiento en la próxima encarnación (Baumann, 2001).

"El budismo modernista o reformista comienza a fines del siglo XIX cuando líderes budistas comenzaron a responder a los estímulos causados por la modernización, lo que incluyó un énfasis en elementos racionales, un creciente uso de textos sagrados, un renovado énfasis en la meditación y un hincapié en la reforma social y el universalismo, todo ello de la mano de una eliminación tácita de la cosmología tradicional. Hay que señalar que el budismo tradicionalista no desapareció con la llegada del reformista, sino que ambas corrientes coexistieron en medio de mutuas críticas. Una de las influencias que incidió en el desarrollo del budismo modernista es la presencia colonial, ya que muchos occidentales en el siglo XIX y XX manifestaron un gran interés en las tecnologías meditativas. Una institución occidental que ha vertebrado gran parte de estas inquietudes a inicios del siglo pasado, y que por lo tanto ha tenido un rol medular no solo en la difusión de las prácticas y filosofías orientales, sino también en la reformulación de las mismas en Asia, ha sido la Sociedad Teosófica (Sharf, 1995; Baumann, 2001).
} 
internacional Vipassana Meditation, que aquí nos ocupa (Sharf, 1995). En el próximo apartado haremos un breve recorrido por la trayectoria vital de los precursores y fundadores de esta organización, lo cual nos permitirá luego realizar una serie de reflexiones acerca de los principales rasgos que configuraron la forma actual de esta organización.

\section{EL LINAJE DE LA MEDITACIÓN VIPASSANA}

Según Pranke (2010), fue el monje Medawi (1728-1816) quien escribió los primeros manuales de meditación vipassana de los cuales se tienen registro en Birmania. Pero el promotor más significativo de esta práctica en la modernidad fue Ledi Sayadaw, nacido en Birmania en 1846. Ledi Sayadaw ingresó como novicio a un monasterio budista desde temprana edad y fue ordenado monje a los 20 años. En 1867 se mudó a la que era por entonces la capital del país, Mandalay, y estudió con los más célebres monjes y eruditos de la época. Fue un excelente estudiante de los textos budistas, lo cual lo habilitó para ejercer más tarde como profesor del Canon Pali. En 1871 participó del ya mencionado V Concilio Budista, colaborando con la edición y traducción de los textos del Abhidhamma, un importante texto del Canon Pali. En 1882 se mudó a Monywa, donde comenzó a practicar meditación vipassana en una pequeña ermita. En 1886, un año después de la invasión y conquista británica, se retiró a meditar al bosque de Ledi, al norte de Monywa. Al año siguiente, fundó un monasterio en esa localidad para dar cobijo a los monjes (bhikkhus) de distintas partes de Birmania que acudían a recibir sus enseñanzas sobre meditación y a formarse en filosofía budista.

En 1911 recibió un doctorado honoris causa en literatura por la Universidad de Rangún. Durante todo este tiempo sostuvo una prolífica actividad como escritor, componiendo poemas, ensayos, cartas y manuales de budismo publicados en lengua pali y birmana. En ocasiones, dejaba su monasterio y viajaba por todo el país dando clases de filosofía budista y enseñando vipassana en varios centros fundados por sus estudiantes. Su deseo era que los laicos sin formación especializada pudieran comprender el budismo y practicar meditación. Gracias a sus viajes y a sus escritos, tuvo una influencia seminal en la revitalización de la práctica de la meditación en el seno de la sociedad secular. Murió en 1923 a los 77 años de edad. 
Uno de los más destacados discípulos de Ledi Sayadaw fue Saya Thetgyi, un maestro de meditación laico que nació en 1873 en un pueblo rural al sur de Rangún. Tuvo una escasa educación escolar y una infancia ardua, ya que sus padres eran pobres - no poseían tierras ni cultivos - y trabajó desde pequeño en la manutención de la familia. A los diez años su padre murió, lo que sumió a su madre - quien era vendedora de comida ambulante - $\mathrm{y}$ a sus tres hermanos en una situación aún más precaria. Pero a los 16 años su situación mejoró, ya que contrajo matrimonio con la hija de un rico terrateniente y comerciante de arroz. Hasta los 30 años tuvo una próspera y feliz vida rodeado de su esposa, sus tres hijos y numerosos parientes y allegados.

En 1903 una epidemia de cólera azotó la aldea, llevándose la vida de dos de sus hijos y la de muchos otros familiares. Esta calamidad fue un punto de inflexión en su biografía, ya que lo motivó a abandonar su hogar para profundizar su búsqueda espiritual, ya que anteriormente, a los 23 años, había comenzado a practicar meditación con un maestro laico, Saya Nyunt. Viajó por todo el país para estudiar y meditar con distintos sabios budistas. Finalmente, siguiendo el consejo de su primer instructor, Saya Nyunt, fue a visitar a Ledi Sayadaw a su monasterio en Monywa. Se quedó allí por siete años realizando grandes progresos en su camino espiritual.

Tras regresar a su tierra natal de Rangún en 1910, permaneció recluido en meditación en un cuarto emplazado en las adyacencias de su anterior hogar, sin volver a la vida de padre de familia, aunque recibía alimentos de parte de sus parientes. Por pedido de Ledi Sayadaw en 1914 comenzó a enseñar a algunos de los lugareños que comenzaron a afluir a su lugar de retiro. En los siguientes treinta años su fama no dejó de crecer, y contó entre sus estudiantes a todo un amplio espectro de personas, desde obreros y campesinos hasta monjes y eruditos doctos en filosofía budista. Falleció en 1945 a los 72 años de edad una noche en la que estaba meditando acompañado de sus discípulos.

Uno de sus estudiantes fue Sayagyi U Ba Kin, que nació en 1899 en Rangún, antigua capital de Birmania, en el seno de una familia obrera. Destacado en todas las asignaturas, logró una beca para estudiar el secundario en la elitista St. Paul School, siendo siempre el primero de la clase. Allí aprendió inglés, lo cual aumentó sus posibilidades de conseguir un trabajo en el gobierno colonial de Inglaterra. Tras sus estudios secundarios, en 1917 comenzó a trabajar, primero como periodista en un diario, y luego como funcionario de la Auditoría General de Birmania, aprobando el examen de contador en 1926. Estaba casado y tenía seis hijos, cinco varones y una mujer. En 1948, cuando 
Birmania logró la independencia de la India, fue nombrado Auditor General de la Nación. Las dos décadas siguientes se desempeñó en varios cargos directivos en diversos departamentos del estado socialista birmano.

Sayagyi conoció la meditación en 1937 cuando realizó un curso de 10 días con Saya Thetgy. Cuatro años más tarde conoció a Webu Sayadaw, un reconocido monje que otorgaba la mayor importancia a la práctica de la meditación vipassana. Fue él quien lo exhortó a que enseñase a sus conocidos este camino espiritual, aunque no fue hasta una década más tarde que se dedicó a ello con mayor énfasis. Con el tiempo se fue convirtiendo en un reconocido maestro de meditación laico con un estilo que, al igual que sus predecesores, hacía hincapié en la experiencia directa del vipassana por sobre el estudio académico. Con frecuencia, los empleados a su cargo tomaban cursos de meditación con él, lo cual lo condujo a fundar, en 1950, la Asociación Vipassana de la Auditoria General.

En 1952 fundó en Rangún el International Meditation Center y entre 1954 y 1956 participó a activamente como miembro organizador y contador en el VI Concilio Budista llevado a cabo en Rangún. Gracias a su fluido conocimiento de la lengua inglesa, su centro adquirió un fuerte carácter internacional ya que contaba entre sus estudiantes a budistas occidentales, académicos, diplomáticos, indios, y otros extranjeros provenientes de diversas partes del mundo ${ }^{12}$.

U Ba Kin combinó durante toda su vida sus responsabilidades laborales, la vida familiar y la enseñanza del vipassana. Trabajo en el gobierno hasta los 67 años, cuatro años antes de su muerte, y era considerado como alguien que se destacaba por su integridad moral y su eficiencia en el desempeño de sus funciones. No sólo era conocido por su honestidad, sino también por ser generoso, austero, estricto con la disciplina, paternalmente bondadoso, paciente y trabajador ${ }^{13}$.

Si bien siempre anheló viajar a EEUU y a la India para enseñar vipassana, debido a las restricciones del gobierno birmano nunca pudo salir del país. Además, sus obligaciones

\footnotetext{
${ }^{12}$ Una de sus estudiantes fue la reconocida socióloga de la religión británica Elizabeth Nottingham, quien publicó en un artículo académico con sus impresiones tras la realización del curso de 10 días en 1958 (Nottingham, 1960).

13 Se cuenta que, cuando los funcionarios británicos huían ante el bombardeo japonés en 1942, encontró entre los escombros la caja fuerte de la estación de ferrocarriles de Rangún en la que trabajaba como auditor. Retiró el dinero y, en lugar de quedárselo para sí mismo - o entregárselo a los ocupantes japoneses -, viajó todo un día en tren para devolverlo a los funcionarios británicos antes de que huyeran del país. Cuando fue nombrado presidente de la Cámara de Comercio Agrícola de Birmania, se destacó por limpiar de corrupción dicho importante organismo estatal, no aceptando nunca las dádivas y sobornos que a menudo recibía.
} 
laborales limitaban el tiempo que podía dedicarle a la enseñanza, aunque creía que era tiempo de que sus discípulos difundan el vipassana a un público más amplio. Antes de su muerte nombró maestros de diferentes países para que lleven su mensaje.

Uno de ellos fue Satya Narayan Goenka, quien nació en Mandalay en 1924. Pertenecía a una familia acaudalada de origen indio, miembros de la etnia marwari, conocida por su larga tradición como comerciantes e industriales. Sus padres profesaban el hinduismo en su vertiente más conservadora. Casado y padre de una familia numerosa, desde joven Goenka se convirtió en un exitoso hombre de negocios y líder de la comunidad india en Birmania. Sin embargo, su placentera existencia se vio perturbada cuando a los 26 años comenzó a padecer migrañas severas que lo debilitaban y lo obligaban a emplear morfina para soportar el dolor. Viajó por todo el mundo visitando a los mejores médicos para tratar su dolencia y evitar caer en una incipiente adicción a esta potente droga, pero ninguno de los especialistas consultados pudo ayudarlo. Por indicación de un amigo, quien sospechaba que su malestar podía tener una etiología psicosomática, Goenka fue a hacer un curso de 10 días de meditación vipassana con Sayagyi U Ba Kin en 1955. Sus dolores desaparecieron y experimentó una profunda experiencia espiritual que le dio un vuelco radical a su vida.

Practicó junto a su maestro por catorce años, hasta que aquel lo autorizó a enseñar meditación en 1969. Ese mismo año viajó a la India con un permiso especial para visitar a su madre, quien se encontraba enferma, y también para cumplir el deseo de su maestro de llevar el vipassana a su país de origen. Tras su llegada, encontró serias dificultades para organizar el primer curso ya que conocía a pocas personas y la mayoría de sus parientes desaprobaban el vipassana debido a que divergía de su religión tradicional: el hinduismo. Sin embargo, la resistencia cedió en parte, y finalmente el primer curso se pudo organizar con la participación del padre y la madre de Goenka, junto a unos pocos parientes y conocidos. A partir de este momento, la práctica del vipassana fue adquiriendo cada vez mayor pregnancia entre su círculo social, y muchos cursos de 10 días se organizaron con personas que concurrían por recomendación de familiares y amigos. En 1976 fundó Dhamma Giri, su primer centro de meditación, situado en la India, y rápidamente otros centros se fueron estableciendo por todas las regiones de este país.

Muchos estudiantes occidentales comenzaron a asistir a los cursos de Goenka en la India desde mediados de los años 1970, por lo cual el gurú birmano empezó a recibir frecuentes invitaciones a enseñar en los países de donde estos estudiantes provenían. Por 
su parte, Goenka estaba dispuesto a viajar pues, ya bien establecido el dhamma (doctrina budista) en la India, deseaba cumplir el deseo de su maestro U Ba Kin de llevar la práctica del vipassana al resto del mundo. La motivación para cumplir esta misión se vio acrecentada cuando algunos estudiantes estadounidenses que habían tomado unos pocos cursos en la India abrieron un centro en su país natal. Goenka sintió el peligro de que su enseñanza perdiera su pureza debido a que aquellos estudiantes no estaban autorizados para enseñar. Pero el pasaporte no le permitía viajar fuera de la India, aun cuando realizó una solicitud personal al presidente del gobierno socialista birmano, $\mathrm{Ne}$ Win, al cual lo unía una fluida amistad. La única solución era renunciar a la nacionalidad birmana y aceptar la india, habilitándolo así a obtener un pasaporte válido para viajar a todo el mundo. Esta era una decisión difícil de tomar ya que, aunque la India era la tierra de sus antepasados, Birmania había sido el país que lo vio nacer.

Tras meditarlo varios años, en 1979, diez años después de su llegada a la India, Goenka se decidió a esparcir su enseñanza al resto del mundo, renunciando a su ciudadanía birmana. Ese mismo año condujo los primeros cursos de 10 días en Francia, Canadá e Inglaterra. En 1985 fundó el Vipassana Research Institute en Dhamma Giri, el cual tenía como objetivo la investigación de las fuentes históricas y la aplicación de la técnica de vipassana en la vida cotidiana. Los resultados principales han sido la traducción y publicación del Canon Pali completo en soporte digital y la edición de libros y artículos escritos por U Ba Kin, Goenka y otros autores vinculados al movimiento' ${ }^{14}$.

Goenka fue un prolífico escritor y orador, llegando a hablar incluso en la Asamblea General de las Naciones Unidas y en el World Economic Forum de Davos. A lo largo de su vida condujo cerca de 300 cursos de meditación de 10 días y nombró unos 1200 profesores asistentes habilitados para representarlo en el dictado de estos cursos desde el año 1982. Falleció en la India en el año 2013 a los 90 años.

Recapitulando el recorrido anterior podemos señalar que Ledi Sayadaw fue un pionero en incorporar el entrenamiento meditativo a la formación intelectual, armonizar la teoría con la práctica. Al mismo tiempo, tuvo un rol seminal a la hora de posibilitar la práctica de la meditación a los laicos. Anteriormente se consideraba que había que desarrollar completamente los estados extáticos de absorción contemplativa (jhanas)

\footnotetext{
${ }^{14}$ Actualmente el Vipassana Research Institute publica un boletín trimestral internacional, ofrece material audiovisual con los numerosos discursos de Goenka y organiza un programa de estudios de lengua pali para estudiantes avanzados de vipassana.
} 
mediante la concentración en la respiración [anapanasati] antes de practicar la observación de la impermanencia (anicca) de las sensaciones corporales y los procesos mentales (vipassana). Pero para alcanzar estos estados de concentración profundos se requería de la reclusión por grandes períodos de tiempo en entornos monásticos ${ }^{15}$.

Con el fin de poder insertarse en la sociedad, se dio una simplificación de la práctica y un cambio en la metodología espiritual que posibilitó la participación de los seglares, rasgo fundamental en el Movimiento Vipassana. de ahora en más éstos ya no estaban limitados a practicar la moralidad, la caridad y la devoción a fin de crear las condiciones para liberarse en futuras vidas. La figura de Saya Thetgyi da testimonio de ello, la cual se destaca por romper las convenciones de la época al convertirse en el primer maestro laico de vipassana. De forma que, si la figura de Ledi fue un hito en la integración de la dimensión teórica con la práctica, la de Thetgyi representa la plena ruptura de las fronteras que anteriormente existían entre la esfera monástica y laica en la división de las actividades religiosas. No obstante, si bien Saya fue un maestro laico, después de dedicarse a la meditación su vida no fue la de un padre de familia convencional. Aunque su centro de meditación se ubicaba en las tierras de sus parientes, Saya no volvió al hogar matrimonial. En este sentido, es posible señalar a U Ba Kin como el primer referente que con su vida da testimonio de la posibilidad de integrar la vida familiar y laboral con la vida religiosa y meditativa. También dio el puntapié inicial para la difusión global del vipassana ya que, como mencionamos, a sus cursos acudían numerosos extranjeros. Asimismo, fue él quien comenzó a enseñar vipassana con una pedagogía y una terminología que lo hacía más accesible a todo tipo de público.

De todas formas, hay que mencionar que, desde la perspectiva de Goenka, la enseñanza de U Ba Kin no sería un nuevo método, sino el resurgimiento de un método tradicional, auténtica enseñanza esencial del Buda:

En su técnica, U Ba Khin no efectuó ni la más mínima desviación de las enseñanzas del Buddha, no obstante, después de incesante investigación práctica y experimentación, desarrollo instrucciones propias, más apropiadas a las demandas de los tiempos modernos (...) los resultados y descubrimientos de estos estudios,

\footnotetext{
15 Según menciona Goenka (2011), tradicionalmente, vipassana se enseñaba en retiros de siete semanas de duración. Con la entrada del siglo XX, los profesores de esta tradición empezaron a experimentar con períodos más cortos para adecuarse al ritmo de vida más acelerado. Intentaron treinta días, dos semanas, diez días, hasta reducirlo a siete días, y constataron que un período más corto que diez días no resulta suficiente para que la mente entre en calma y pueda trabajar con profundidad.
} 
llevados a cabo en una sala de meditación especial en la oficina de la Auditoría General, permitieron que Sayagyi presentara a los laicos el Dhamma de Buddha de una manera sistemática y científica, atractiva para la mente moderna [Goenka, 2011, p. 28-29).

En cierta forma, U Ba Kin cumplió un rol fundamental en la democratización de la meditación en Birmania desde mediados del siglo pasado, integrando plenamente la esfera sagrada con la secular, mientras que Goenka le dio a esta tradición un alcance global, integrando Oriente con Occidente, contribuyendo a la formación del denominado "budismo global o postmoderno" (Baumann, 2001) ${ }^{16}$. Creemos que gran parte de su éxito se debe al hábil manejo de ciertos topos fundamentales y estrechamente interrelacionados, los cuales podemos sintetizar en una postura crítica a la religión, la apelación a la cientificidad del vipassana, la narrativa del tesoro espiritual y la importancia otorgada a la vida cotidiana. En otras palabras, es posible afirmar que la difusión de la organización Vipassana Meditación en todo Occidente pueda explicarse por la puesta en juego de ciertos temas vinculados con la secularización, el racionalismo, la autenticidad y la ética intramundana. A continuación examinaremos cada uno de estos puntos con cierto detalle.

\section{PRINCIPALES RASGOS DEL DISCURSO DE VIPASSANA MEDITATION}

La primera característica del discurso de la organización fundada por Goenka es su carácter secularizado y universalista, el cual involucra muchas veces una crítica al condicionamiento religioso recibido, al ritualismo y el afán de conversión. Así, se sitúa al dhamma como una ley universal que va más allá de las verdades parciales, contingentes y particulares de las religiones. En este contexto, se dice que la verdadera sabiduría de la enseñanza budista no requiere ninguna creencia "sectaria" o filosófica. Por ejemplo, la atención a la respiración, propia de la misma práctica de la meditación, se encuentra por fuera de cualquier división religiosa o de otra índole, ya que uno "no puede decir que la respiración sea hindú, cristiana, budista o musulmana". O al prestar atención a las sensaciones y las emociones, uno "no puede decir que le ira, el calor, el dolor, la picazón

16 Según Baumann (2001), el budismo global o postmoderno se desarrolla mediante un proceso de secularización, psicologización, hibridización y globalización del budismo modernista y su membrecía se compone principalmente de individuos sin antepasados orientales. 
o la tristeza sean occidentales, orientales, femeninas o masculinas". De modo que el vipassana "ofrece beneficios a todos los que la practican, sin que intervengan condicionamientos basados en la raza, case social o sexo"..."en un país marcadamente dividido todavía por castas y religiones" (Hart, 2010, p. 13).

Si bien se manifiesta una abierta tolerancia a todas las religiones, se enfatizan los peligros del ritualismo, el sectarismo y la conversión. Al no ser una religión, ni siquiera identificarse como budismo, el vipassana "no intenta convertir gente de una religión organizada a otra". El propio Goenka cuenta el alivio que sintió cuando U Ba Kin le dijo que no necesitaba convertirse en budista para participar de su primer curso, al cual había dudado de asistir debido a que era un hindú ortodoxo. Consiguientemente, si bien los rituales religiosos son tolerados, se consideran como meras formas vacías de contenido, parte de un condicionamiento que uno debe aceptar debido al nacimiento en determinada cultura.

El segundo rasgo central de la cosmovisión vipassana es el racionalismo, que implica la afirmación de la naturaleza científica del dhamma y la crítica al conocimiento teórico sin la experiencia de la verdad. Así, uno de los temas recurrentes del discurso de Goenka es la noción de que la práctica del vipassana y la doctrina budista que lo sustenta es científica: reclama que el Buda es "un súper-científico" cuya enseñanza de paz y armonía es particularmente relevante para atravesar la crisis del mundo contemporáneo. Al mismo tiempo, se considera que la meditación vipassana es "lógica", "racional", "simple", "experimental", con "resultados concretos". Enfatiza Goenka: "Definitivamente, insuperable e incomparable es la práctica del Dhamma. ¡Tan fácil! ¡Tan clara! ¡Tan científica y beneficiosa!" (Goenka, 2011, p. 23-26).

En este contexto se resalta la idea del Buda de no aceptar algo porque otro lo dice, sino porque uno lo ha verificado por sí mismo, mediante un proceso de experimentación de primera mano. Se diferencia entre comprensión teórica o intelectual [pariyatti) y comprensión práctica o experiencial (paripatti). La comprensión teórica es útil para acercar a las personas a la práctica de la meditación o para resolver sus dudas una vez que la han comenzado.

Al mismo tiempo, se centran los esfuerzos en traducir la doctrina empleando un lenguaje en armonía con los conceptos científicos modernos. Por ejemplo, se destaca una serie de concordancias entre el modelo científico y las ideas budistas. A nivel cosmológico, se relacionan la teoría del Big Bang con los ciclos de contracción y expansión del universo 
de la cosmogénesis budista. Además, la ley de la interdependencia se vincula con reflexiones sustentadas por la astronomía y la química tales como el postulado de que somos energía solar - proveniente de átomos y estrellas -, transformada en vida, sujeta a la ley de la entropía que señala que todas las estructuras compuestas se disuelven. A nivel biológico, se argumenta, por ejemplo, que el término "anicca", es decir "interdependencia", está firmemente enraizado en la biología, ya que a cada segundo se producen millones de reacciones químicas y energéticas sin cesar. Finalmente, las frecuentes referencias a la física cuántica resaltan que las observaciones realizadas por esta disciplina en cuanto a la equivalencia entre materia y energía, la dualidad de partícula y onda, la resonancia a distancia de los electrones y la no-división entre el observador y lo observado, entre otras cosas, en cierta forma "re-descubren", a la vez que confirman y autentifican, las observaciones introspectivas realizadas hace 2.500 años por el Buda: el mundo material consiste en vibraciones surgiendo y desapareciendo y no existe un "yo" real y permanente a nivel psicológico, sino fenómenos mentales (pensamientos, sensaciones, emociones] que también surgen y desaparecen, sin una identidad que los unifique.

Por otra parte, el tercer rasgo fundamental de la narrativa del vipassana es el fuerte acento en una ética intramundana que le otorga la mayor importancia a la vida laica y comprometida con la sociedad. Se pone en primer lugar la vida de familia, las responsabilidades familiares, la necesidad de obtener el sustento material tanto para uno mismo como para los que dependen de uno y que implican la participación activa en los asuntos mundanos. Desde esta perspectiva, la práctica de vipassana es una tecnología de cambio social que permitiría transformar el mundo secular, signado por el odio, la ansiedad, el miedo, la corrupción, la amoralidad y el materialismo. El impacto en la vida cotidiana estaría dado por la capacidad del vipassana de generar moralidad, ecuanimidad, mayor energía, paz y armonía en cada individuo, y a que estos efectos beneficiosos se extiendan al resto de la sociedad ${ }^{17}$.

\footnotetext{
${ }^{17}$ En los discursos y textos de los referentes de esta organización se recupera con frecuencia el ejemplo de Sayagyi U Ba Kin, quien como referimos tuvo una intensa actividad como funcionario público, la cual da testimonio de la perfección moral derivada de su compromiso con el dhamma, o se resalta la trayectoria de Goenka como industrial y hombre de negocios, considerado como una "una persona pragmática, que está en contacto con las dificultades de la vida ordinaria y que es capaz de afrontarlas con agudeza, manteniendo una extraordinaria calma mental en cualquier situación" (Hart, 2010, p. 13-14). También se destaca a menudo que ambos maestros siempre fueron hombres de familia, con muchos hijos, esposa y responsabilidades domésticas. Goenka menciona a menudo que el hecho de presentarse en público siempre con su esposa es testimonio de su vida como seglar.
} 
Conjuntamente, existen algunos elementos normativos que favorecen la ética intramundana del vipassana, previniendo la diferenciación entre una esfera seglar y otra estrictamente espiritual. Por ejemplo, uno de ellos es el requisito de que los profesores designados por Goenka para representarlo tengan su propia fuente de ingresos. Ellos no pueden recibir ningún pago, donación o beneficio por realizar el "servicio de enseñar", y solamente lo deben hacer para recibir "la satisfacción de ver la felicidad de las personas al final de los diez días". Otro elemento normativo es la imposibilidad explícita de residir en los centros permanentes del grupo por períodos extensos de tiempo.

Finalmente, el cuarto topos que traspasa continuamente el discurso vipassana es el que hace referencia a la autenticidad: el vipassana es la esencia de la enseñanza del Buda. Se dice que esta práctica ha sido tradicionalmente mantenida por unas pocas personas, ya que la mayor parte de la comunidad monástica se inclinó por el estudio y la enseñanza del Canon Pali, por el conocimiento intelectual (pariyatti), sin darle demasiada importancia a la práctica experiencial (patipatti). Esta situación habría generado la decadencia del budismo en la India, e incluso propiciado que la enseñanza intelectual también se pierda. De ahí la importancia de transmitir el vipassana sin adulteraciones, ya que cualquier cambio conllevaría irremediablemente a la pérdida de este saber. Esta tarea ha sido realizada por una cadena continua de maestros que, generación tras generación, mantuvieron la técnica de vipassana en su prístina pureza hasta la actualidad.

En este contexto, se considera que Birmania jugó un papel clave en mantener el dhamma puro, en custodiar la enseñanza durante milenios posibilitando su resurgimiento en tiempos modernos. Según afirma Goenka, el vipassana fue enseñado primero por Bhallika y luego por Gavampati en Birmania, pero tres siglos después fue contaminado. Luego, en tiempos de Asoka, Sona y Uttara recuperaron la pureza del dhamma enseñando la técnica de vipassana en el sur del país, preservada, como señalamos por una cadena de maestros y discípulos por siglos. De ahí que Goenka refiere continuamente la gratitud que debemos sentir hacia esta cadena de transmisores de vipassana que posibilitaron que la enseñanza se mantenga inalterada y que, 2.500 años después, pueda ser recibida por el mundo entero. En suma, se considera a Birmania como un lugar sagrado, un refugio que mantuvo y guardo para la posteridad no solo las palabras de Buda, sino también su enseñanza práctica, transmitida a través de un linaje de maestro y discípulo en toda su pureza. Durante dos mil años la técnica de vipassana, la cual se considera la esencia de la enseñanza del Buda, se mantuvo allí. Además, se considera que Birmania debe "reparar su 
deuda con la India" por haber recibido el dhamma, y la forma de hacerlo es que la "invaluable joya de vipassana vuelva a enseñarse en el país que la vio nacer hace más de 2500 años" (Goenka, 2011, p. 10)

En estrecha relación con lo anterior, el componente profético, milenarista y restaurador con el que Goenka presenta el vipassana es central. Su maestro U Ba Kin y sus antecesores afirmaban una antigua profecía que decía que, 2.500 años después de la muerte del Buda, la enseñanza resurgiría y se difundiría por todo el mundo. Esto daría inicio a la "era del segundo sasana", un nuevo período de difusión de las ideas del budismo. Así, los años 1960 son identificados con el cumplimiento de ese término, por lo cual U Ba Kin solía decir: "la hora de vipassana ha Ilegado"m8. Además, afirmaba que la India estaba lista para aceptar rápidamente su tesoro perdido, ya que mucha gente había nacido con la suficiente cantidad de méritos como para poder recibir el vipassana. Según refiere Goenka, su maestro lo "eligió para plantar las semillas del dhamma alrededor del mundo" [Goenka, 2011, p. 11], y predijo que a su llegada a la India recibiría una señal que le indicaría su éxito. Este augurio se habría cumplido cuando, al descender del avión que lo llevó de Rangún a Calcuta, un terremoto sacudió todo el norte de la India: "el país estaba emocionado de recuperar la joya del dhamma perdida hace tanto tiempo", declaró Goenka. Pese a los obstáculos que tuvo en sus comienzos como difusor del vipassana en la India, el explosivo crecimiento en el número de cursos y de centros que fundó en aquel país y en el resto del mundo en las décadas posteriores es interpretado como el efectivo cumplimiento de esta profecía.

\section{REFLEXIONES FINALES}

Tras nuestro recorrido podemos observar que el budismo no se difundió mediante un proceso unidireccional que, tal como las ondas se alejan de un centro cuando una piedra es lanzada a un lago, irradió desde su emplazamiento originario en la India hacia el Lejano Oriente y el Sudeste Asiático. No solo el desarrollo sino también, en ocasiones, la misma supervivencia del budismo implicó el flujo y reflujo de personas, ideas, prácticas y textos. Este tránsito configuró múltiples vías de doble mano que conectaron lugares como Ceilán, Birmania, Tailandia y el norte de la India, los cuales en diferentes momentos

${ }^{18}$ De hecho, el libro de Goenka (2011) citado en este trabajo se inspira en esta expresión para su título. 
históricos fueron tanto centros consolidados de enseñanza como lugares en los cuales el budismo se perdió. Por ejemplo, en los últimos cinco siglos el budismo tuvo varios momentos en los cuales decayó en Ceilán, por lo cual debió ser revitalizado mediante la invitación de monjes y eruditos de Birmania y Tailandia por parte del rey de Ceilán para que reformaran la comunidad monástica de su país. Asimismo, el budismo también fue reintroducido en el subcontinente indio en el siglo XVI desde Birmania, donde había desaparecido debido a la rivalidad con el hinduismo y a la expansión musulmana en su territorio. De modo que aquí tenemos varias vías de doble mano por las cuales el budismo no solo fluye desde Asia Meridional hacia el Extremo Oriente, sino que también circula en sentido inverso en diferentes momentos históricos, proceso que ha favorecido su enraizamiento en todo este territorio.

Este trabajo ha explorado especialmente una de estas vías de doble mano, analizando el tránsito de agentes, saberes y estructuras simbólicas budistas entre la India, Birmania y Occidente, centrándose en la forma en que esta circulación dio origen a una de las organizaciones budistas más difundidas en Occidente: la Vipassana Meditation. Tanto las biografias de Ledi Sayadaw, Saya Thetgyi, Sayagi U Ba Kin y S. N. Goenka, como los principales temas del discurso del vipassana referidos en el apartado anterior recapitulan de alguna forma los desafíos que tuvo que afrontar y los escollos que tuvo que superar el desarrollo histórico del budismo modernista: la integración entre teoría y práctica, entre la esfera consagrada y la laica, entre la vida de familia y la práctica religiosa, entre el budismo y la ciencia, entre la tradición y la modernidad, entre Oriente y Occidente. En suma, los principales rasgos de la narrativa vipassana pueden leerse como una crítica al budismo tradicionalista realizada desde la óptica del budismo reformista, el cual a su vez surgió bajo el influjo de la modernidad occidental. Al mismo tiempo, el discurso vipassana da cuenta de cómo la construcción de un budismo global o postmoderno puede involucrar la puesta en juego de una innovación conservadora, la vuelta a los orígenes de un budismo inicial que pone en primer lugar la práctica de la meditación.

En el año 2009 Vipassana Meditation finalizó la construcción de la Global Vipassana Pagoda en Bombay. Su forma reproduce la famosa pagoda de Shwedaggon de Birmania $y$, al igual que ella, resguarda algunas reliquias del Buda en su interior. El salón principal, con capacidad para albergar 8000 meditadores, está coronado por el mayor domo de piedra sin pilares que lo sostenga del mundo, con un diámetro de casi 100 metros. Este edificio, que combina la tecnología más avanzada con un diseño milenario, 
simboliza la gratitud de Birmania y su comunidad budista a la India, lugar de origen del Buda y su enseñanza. Asimismo, y desde una mirada descentrada del discurso nativo, podemos señalar que este monumento representa no sólo la relación entre estos dos países, sino también la integración de Oriente con Occidente, el budismo con la ciencia, la tradición con la modernidad, la racionalización y secularización que caracteriza a los movimientos religiosos transnacionales. No es casual que el mismo nombre de esta pagoda, "diseñada para durar mil años", no incluya el término "budismo": es la "Global Vipassana Pagoda", testimonio de un budismo global que se trasciende a sí mismo, formado por la concurrencia de fenómenos que atraviesan el Lejano Oriente, la India y Occidente.

\section{REFERENCIAS BIBLIOGRÁFICAS}

BAREAU, André. El Budismo en Ceilán y en Asia Sudoriental. In: PUECH, Henri-Charles (dir.) Historia de las religiones: Las religiones constituidas en Asia y sus contracorrientes II. Vol. 10, p. 1-30. Madrid: Siglo XXI, 1981.

BAUMANN, Martin. Buddhism in Europe: Past, Present, Prospects. In: PREBISH, Charles S. and Martin BAUMANN. Westward Dharma: Buddhism Beyond Asia. California: University of California Press, 2002.

BAUMANN, Martin. Global Buddhism: Developmental Periods, Regional Histories, and a New Analytical Perspective. Journal of Global Buddhism, n. 2 , p. 1-43, 2001.

CARVAlHO, António Manuel Simões Lopes Paiva. Performing Meditation: Vipassana and Zen as Technologies of the Self. Tesis doctoral. University of Exeter, 2013.

EDDY, Glenys. The Vipassana Retreat Experience: A Consideration of the Meditation Retreat as a Religious Paradigm of Travel. Literature \& Aesthetics, n. 22 (1), p. 38-59, 2012. GOENKA, Satya Narayan. El tiempo de vipassana ha Ilegado. Buenos Aires: Editorial Metta, 2011.

HART, William. El arte de vivir. Buenos Aires: Editorial Metta, 2010.

HARVEY, Peter. El Budismo. Cambridge: Cambridge University Press, 1998.

NOTTINGHAM, Elizabeth. Buddhist Meditation In Burma. Rangún: International Meditation Centre, 1960.

PAGIS, Michal. Evoking Equanimity: Silent Interaction Rituals in Vipassana Meditation Retreats. Qualitative Sociology, n. 38, p. 39-56, 2015.

PAGIS, Michal. Producing intersubjectivity in silence: An ethnographic study of meditation practice. Ethnography, n. 11 (2), p. 309-328, 2010. 
PRANKE, Patrick. On saints and wizards: Ideals of human perfection and power in contemporary Burmese Buddhism. Journal of the International Association of Buddhist Studies, vol. 33, n. 1-2, p. 453-488, 2010.

SHARF, Robert. Buddhist Modernism and the Rhetoric of Meditative Experience. Numen, v. 2, p. 228-283, 1995.

SMITH, Harold. El Budismo. En: JAMES, E. O. (Dir.) Historia de las Religiones. Tomo II. Tercera edición. Barcelona: Vergara, 1963. 